EPJ Web of Conferences 73, 04004 (2014)

DOI: $10.1051 /$ epjconf/20147304004

(C) Owned by the authors, published by EDP Sciences, 2014

\title{
Target and beam-target asymmetry measurements at MAMI
}

\author{
V.L. Kashevarov ${ }^{1,2, a}$ for Crystal Ball at MAMI, TAPS, and A2 Collaborations \\ ${ }^{1}$ Institut für Kernphysik, Johannes Gutenberg-Universität Mainz, 55099 Mainz, Germany \\ ${ }^{2}$ Lebedev Physical Institute, 119991 Moscow, Russia
}

\begin{abstract}
Preliminary data for target and beam-target asymmetry for the reaction $\gamma p \rightarrow$ $\pi^{0} \eta p$ obtained at the MAMI accelerator are presented. The data are compared to different theoretical predictions.
\end{abstract}

\section{Introduction}

Main features of $\pi^{0} \eta$ photoproduction on protons were studied in works [1-6], were the reaction mechanism was explained through the dominance of the $D_{33}$ wave with a small admixture of positive parity resonances and an insignificant Born terms contribution. Further steps toward investigation of this reaction are related to polarisation observables that are especially sensitive to small reaction amplitudes. There are 64 polarization observables for double pseudoscalar meson photoproduction. Different relationships among these observables and their symmetries decrease this value to 15 independent quantities [7]. Some of these were already measured and analyzed [8-12].

In the present work we measured at the first time the target and the beam-target asymmetries. For the totally exclusive five-fold cross section there are two independent transversal target asymmetries $\left(P_{x}\right.$ and $P_{y}$ ) and two independent circular beam-target asymmetries $\left(P_{x}^{\odot}\right.$ and $\left.P_{y}^{\odot}\right)$ [13]. The observables $P_{y}$ and $P_{x}^{\odot}$ integrated over the phase space of two from three final state particles are equivalent to $T$ and $F$ asymmetries of single pseudoscalar meson photoproduction.

\section{Experimental setup and data analysis}

The experiment was performed at the MAMI C accelerator in Mainz [14] using the Glasgow-Mainz tagged photon facility [15]. In the present measurement the longitudinally polarized electron beam with energy of $1557 \mathrm{MeV}$ and polarization degree of $80 \%$ was used. The longitudinal polarization of electrons is transferred to circular polarization of the photons during the bremsstrahlung process in a radiator. The reaction $\gamma p \rightarrow \pi^{0} \eta p$ was measured using the Crystal Ball [16] as the central spectrometer and TAPS [17] as a forward spectrometer. The solid angle of the combined Crystal Ball and TAPS detection system is nearly $97 \%$ of $4 \pi$ sr.

\footnotetext{
ae-mail: kashev@kph.uni-mainz.de
}

This is an Open Access article distributed under the terms of the Creative Commons Attribution License 4.0, which permits unrestricted use, distribution, and reproduction in any medium, provided the original work is properly cited. 


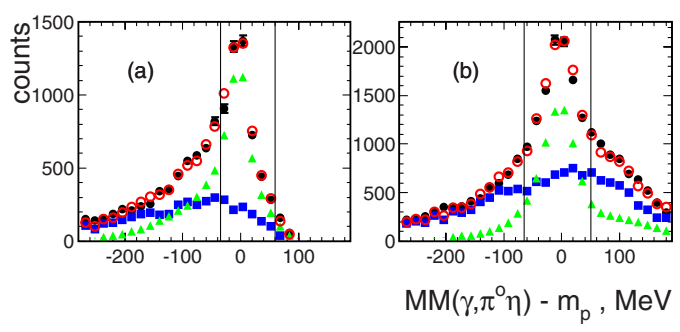

Figure 1. Carbon background subtraction. See text for an explanation.

The experiment requires transversely polarized protons, which were provided by a frozen-spin butanol $\left(\mathrm{C}_{4} \mathrm{H}_{9} \mathrm{OH}\right)$ target. A specially designed ${ }^{3} \mathrm{He} /{ }^{4} \mathrm{He}$ dilution refrigerator was built in cooperation with JINR (Dubna). For transverse polarization a 4 layer saddle coil was installed as the holding magnet, which operated at a field of 0.45 Tesla. The target container with $2 \mathrm{~cm}$ length and $2 \mathrm{~cm}$ diameter was filled by $2 \mathrm{~mm}$ diameter butanol spheres with the filling factor of around $60 \%$. The average proton polarization during beam time period May-June 2010 and April 2011 was 70\% with relaxation times of around 1500 hours. More details about the target are given in Ref. [18].

The event-selection procedure was similar to the one that was described in Ref. [19], where meson pairs were identified via their decay into 2 photons. For the case of 4 detected photons the best solution for the meson pair was found using the $\chi^{2}$ minimization. This was then followed by application of the invariant and missing mass cuts providing good identification of the reaction.

Using the butanol target has an essential disadvantage because of an additional background coming from the reactions on ${ }^{12} \mathrm{C}$ and ${ }^{16} \mathrm{O}$. Detection of the outgoing protons and application of the coplanarity cut suppress the background significantly, but the effect of this procedure is still insufficient. To subtract the residual background events we used the results of the analysis of $\pi^{0} \eta$ photoproduction on the carbon and the liquid hydrogen targets, which were fitted to the butanol data. Because of the magnitude and the shape of the background depend on the initial beam energy and momenta of the final particles, the background subtraction procedure was performed for each bin, where the asymmetries were measured. The procedure of the background subtraction is illustrated on Fig. 1 for two different examples, which are typical for the presented data analysis. Missing mass spectra for the reaction $\gamma p \rightarrow \pi^{0} \eta p$ with the butanol target are shown on Fig. 1a and b by the full black circles. Spectra measured with the hydrogen and carbon targets are presented on the same plots by the green triangles and the blue squares correspondingly. Their absolute values were fitted to the butanol data. The red opened cycles, representing the sum of the hydrogen and carbon contribution, is our result of the fit. To minimize the uncertainties of the background subtraction, the procedure was used only within the missing mass energy interval, which is indicated on the plots by the vertical solid lines.

The systematic uncertainties come mainly from the determination of the proton polarization degree (4\%), circular photon beam polarization degree (2\%), and the background subtraction procedure (3-4\%) and were estimated to be less than $6 \%$. In order to reduce the systematic errors coming from target and detector conditions, the proton polarization direction was regularly reversed during experiment.

\section{Preliminary results}

Figures 2 and 3 show our preliminary results together with different theoretical predictions. The solid and dashed lines are predictions of the isobar model from Ref. [4]. In this model the reaction amplitude contains the sum of $s$-channel Breit-Wigner resonances with the total spin $J \leq 5 / 2$ and the Born terms. Parameters of the resonances were fitted to the experimental data [19]. For the solid line we used the 

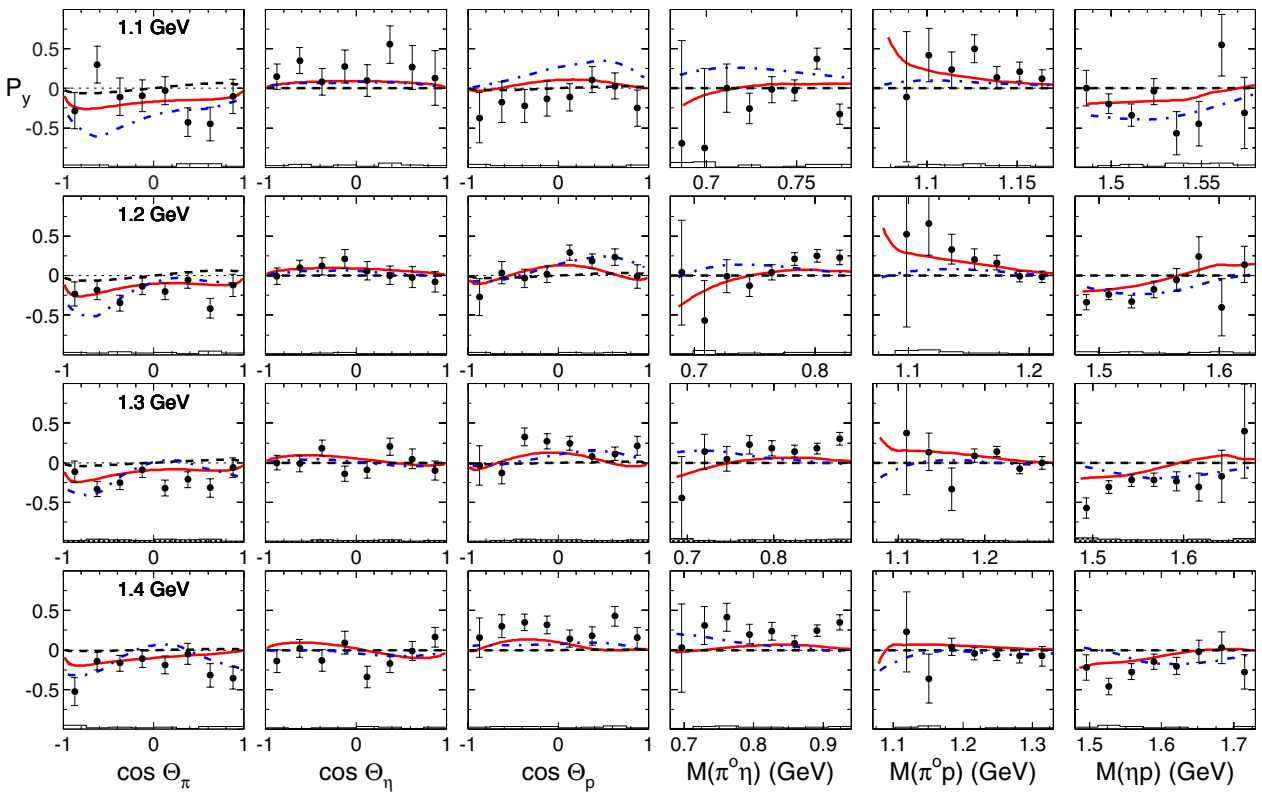

Figure 2. Angular and invariant mass distributions for the target asymmetry $P_{y}$. Our preliminary data are shown by filled circles. Solid curves show the prediction of the isobar model [4]. Dashed curves include only $3 / 2^{-}$wave. Predictions of the Bonn-Gatchina model [5] are shown by dashed-dotted curves. Histograms below are systematic errors. The energy labels on the left panels indicate the central photon energy of $100 \mathrm{MeV}$ bins.
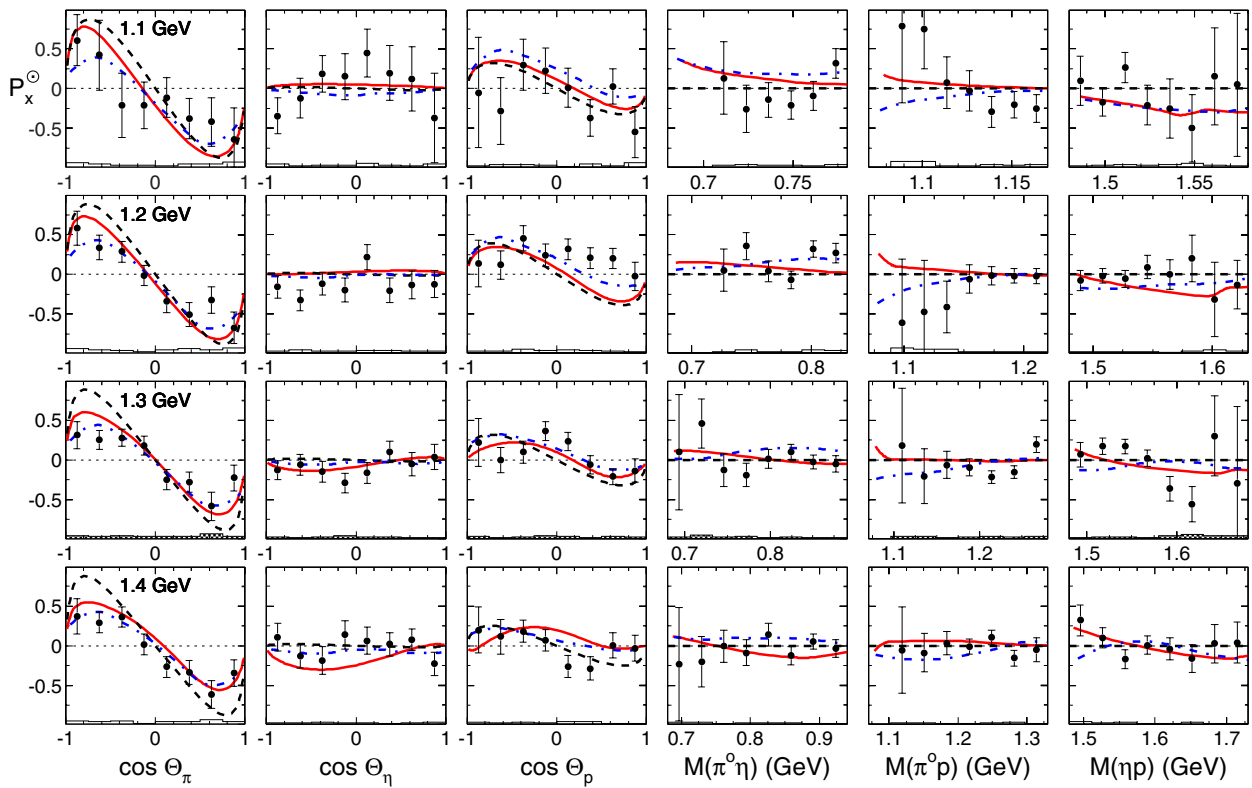

Figure 3. Same as in Fig. 2 for the beam-target asymmetry $P_{x}^{\odot}$. 
fit (I) which gives the best description of the measured linear beam asymmetry $\Sigma[8,9]$. Dashed line include only $\Delta 3 / 2^{-}$resonances.

The dash-dotted line shows predictions of the Bonn-Gatchina multichannel partial wave analysis [5] (solution BG2011-02). Within this approach the positions of resonances, their partial decay widths, and relative strengths are fitted simultaneously to the data sets in different channels, including single and double meson production as well as strangeness production.

As we can see from Figs. 2, 3, both $P_{y}$ and $P_{x}^{\odot}$ demonstrate more complicated behavior, than the one predicted by the single $\Delta 3 / 2^{-}$model. At the same time, the deviation is not large, thus indicating that the role of the states besides $\Delta 3 / 2^{-}$remains restricted. Interference between $\Delta 3 / 2^{-}$and the positive parity states $\Delta 1 / 2^{+}$and $\Delta 3 / 2^{+}$is responsible for the nontrivial angular and energy dependence of all asymmetries presented.

\section{Summary}

Preliminary data for the target and the beam-target asymmetry of the cross section for $\gamma p \rightarrow \pi^{0} \eta p$ obtained with circular polarized photons and transversally polarized protons were presented. The measurements were performed at the MAMI C accelerator using the Crystal Ball/TAPS spectrometer. The polarization observables are sensitive to the contribution of the small components in the reaction amplitude. Obtained data could be usefully for further study of the partial wave content of $\pi \eta$ photoproduction.

This work was supported by the Deutsche Forschungsgemeinschaft (SFB 1044).

\section{References}

[1] M. Döring, E. Oset and D. Strottman, Phys. Rev. C 73, 045209 (2006)

[2] I. Horn et al., Eur. Phys. J. A 38, 173 (2008)

[3] I. Horn et al., Phys. Rev. Lett. 101, 202002 (2008)

[4] A. Fix, V.L. Kashevarov, A. Lee and M. Ostrick, Phys. Rev. C 82, 035207 (2010)

[5] A.V. Anisovich, R. Beck, E. Klempt, V.A. Nikonov, A.V. Sarantsev and U. Thoma, Eur. Phys. J. A 48, 88 (2012)

[6] A. Fix, V.L. Kashevarov and M. Ostrick, Nucl. Phys. A 909, 1 (2013)

[7] W. Roberts and T. Oed, Phys. Rev. C 71, 055201 (2005)

[8] J. Ajaka et al., Phys. Rev. Lett. 100, 052003 (2008)

[9] E. Gutz et al., Eur. Phys. J. A 35, 291 (2008)

[10] E. Gutz et al., Phys. Lett. B 687, 11 (2010)

[11] V.L. Kashevarov et al., Phys. Lett. B 693, 551 (2010)

[12] M. Doring, E. Oset and U.-G. Meissner, Eur. Phys. J. A 46, 315 (2010)

[13] A. Fix and H. Arenhovel, Phys. Rev. C 83, 015503 (2011)

[14] K.-H. Kaiser et al., Nucl. Inst. Meth. A 593, 159 (2008)

[15] J.C. McGeorge et al., Eur. Phys. J. A 37, 129 (2008)

[16] A. Starostin et al., Phys. Rev. C 64, 055205 (2001)

[17] R. Novotny, IEEE Trans. Nucl. Sci. 38, 379 (1991)

[18] A. Thomas, Eur. Phys. J. Special Topics 198, 171 (2011)

[19] V.L. Kashevarov et al., Eur. Phys. J. A 42, 141 (2009) 